Background Cytomegalovirus (CMV) infection is a major cause of morbidity in solid organ transplant recipients. Valganciclovir (ValG), prodrug of the antiviral ganciclovir, is used to prevent or treat CMV infection in this population. is controversial in children, As the percentage of patients reaching the pharmacological target is too low with currently used ValG dosing regimen, our aim was to determine ganciclovir population pharmacokinetics in renal transplant children receiving ValG and propose an appropriate dosage regimen.

Methods After transplantation, children receiving ValG were monitored for plasma ganciclovir concentrations using high performance liquid chromatography. Population pharmacockinetics analysis was performed with NONMEM.

Results 1212 samples from 104 renal transplant patients, aged 2 to 20 years, received ValG to prevent $(n=80)$, treat $(n=12)$, or both prevent then treat $(n=12)$ CMV infection. ValG was administered orally at a daily dose of $19.8 \pm 10.1 \mathrm{mg} / \mathrm{kg}$ (mean $\pm \mathrm{SD}$ ). A two-compartment model with first-order elimination best fitted the data. At steady-state, $\mathrm{AUC}_{24} \mathrm{~h}_{\text {-ss }}$ was $64.5 \pm 23.4 \mu \mathrm{g} / \mathrm{mL} . \mathrm{h}$, apparent clearance $(\mathrm{CL} / \mathrm{F})$ was $0.39 \pm$ $0.14 \mathrm{~L} / \mathrm{h} / \mathrm{kg}$, apparent volume of distribution at steady-state (VDss/F) was $2.4 \pm 0.48 \mathrm{~L} / \mathrm{kg}$. Ganciclovir $\mathrm{CL} / \mathrm{F}$ increased with body surface area, decreased with increasing creatinine concentration and was $15 \%$ higher in boys. Central volume of distribution increased with body surface area (BSA) and showed a $14 \%$ increase in boys. Internal validation was performed.

Conclusion We have successfully built a pharmacokinetic model allowing to propose dosages adapted individually to the characteristics of renal transplanted children.

Disclosure(s) Nothing to disclose

\section{DEFINING SERUM CCL22 AND TREFOIL FACTOR 3 (TFF3) AS PHARMACODYNAMIC BIOMARKERS FOR USE IN A PROOF-OF-CONCEPT CLINICAL TRIAL OF VAMOROLONE IN PAEDIATRIC ULCERATIVE COLITIS}

\begin{abstract}
${ }^{1,2}{ }^{2} \mathrm{LS}$ Conklin*${ }^{3} \mathrm{~A}$ Panigrahi, ${ }^{4} \mathrm{H}$ Gordish-Dressman, ${ }^{2,5} \mathrm{EP}$ Hoffman, ${ }^{5} \mathrm{Y}$ Hathout, ${ }^{2,4} \mathrm{JN}$ van den Anker, 'L Diaz-Calderon. 'Gastroenterology, Hepatology, and Nutrition, Children's National Medical Center, Washington, DC; ${ }^{2}$ ReveraGen BioPharma, Rockville, MD; ${ }^{3}$ Center for Cancer and Immunology Research; ${ }^{4}$ Center for Translational Medicine, Children's National Medical Center, Washington, DC; ${ }^{5}$ School of Pharmacy and Pharmaceutical Science, Binghamton University-SUNY, Binghamton, NY, USA
\end{abstract}

\subsection{6/archdischild-2019-esdppp.39}

Background Paediatric ulcerative colitis (UC) patients would be well-served by a non-steroidal drug to control mucosal inflammation, without long-term and costly commitment to biologics. Vamorolone is a first-in-class alternative to glucocorticoids (GCs), under development for children with Duchenne muscular dystrophy (DMD); preliminary findings demonstrate improved safety compared with GCs ${ }^{1,2}$. We sought to define NFkB-regulated, GC-responsive serum biomarkers for use in a proof-of-concept pilot trial of vamorolone in UC, focusing on TFF3 (produced by intestinal epithelia, GC-responsive in UC), and CCL22 (produced by macrophages, GC-responsive in UC and other inflammatory diseases) ${ }^{3,4}$.

Methods Sera from 10 children with IBD (7 UC, 3 CD) were tested pre and post prednisone/prednisolone $(1 \mathrm{mg} / \mathrm{kg} / \mathrm{day}$, $\max 40 \mathrm{mg}, 1-4$ weeks); 210 proteins responsive to GCs in UC $^{3}$ were analyzed using SOMAscan. Proteins that showed significant change over time were correlated with change in Paediatric Ulcerative Colitis Activity Index (PUCAI) $(\mathrm{p}<0.05$ significance). Percent change in circulating CCL22 was compared with percent change in DMD patients treated with vamorolone $(2 \text { and } 6 \mathrm{mg} / \mathrm{kg} / \mathrm{day}, 2 \text { weeks })^{2}$. Immunoassays were utilized to validate SOMAscan data.

Results CCL22 and TFF3 validated as decreased by GCs in IBD $(p=0.005, p<0.001)$. Decrease in TFF3 correlated with decrease in PUCAI $(r=0.741, p=0.022)$; decrease in CCL22 did not correlate with change in PUCAI. Magnitude of CCL22 decrease in GC-treated UC patients was comparable to that seen in DMD patients treated with $6 \mathrm{mg} / \mathrm{kg}$ of vamorolone $(47 \%$ vs. $33 \%)$. SOMAscan findings in UC validated by immunoassays.

Conclusion Decreases in serum CCL22 likely reflect effect on innate immune response, while decreases in serum TFF3 may reflect intestinal-specific effects of GCs in UC. CCL22 and TFF3, measured by immunoassays, may be useful as objective secondary outcomes reflective of $\mathrm{NFkB}$ inhibition and antiinflammatory activity in a proof-of-concept trial of vamorolone in paediatric UC.

\section{REFERENCES}

1. Hoffman EP, et al. Phase 1 trial of vamorolone, a first-in-class steroid, shows improvements in side effects via biomarkers bridged to clinical outcomes. Steroids. 2018 Jun; 134:43-52.

2. Conklin LS, et al. Phase lla trial in Duchenne muscular dystrophy shows vamorolone is a first-in-class dissociative steroidal anti-inflammatory drug. Pharmacol Res. 2018 Oct; 136:140-150.

3. Heier $\mathrm{CM}$, et al. Identification of pathway-specific serum biomarkers of response to glucocorticoid and infliximab treatment in children with inflammatory bowe disease. Clin Trans/ Gastroenterol. 2016 Sep 15;7(9): e192.

4. Conklin LS, et al. Serum biomarkers of glucocorticoid response and safety in antineutrophil cytoplasmic antibody-associated vasculitis and juvenile dermatomyositis. Steroids. 2018 Dec; 140:159-166.

Disclosure(s) LSC, JNvdA, and EPH are employees of ReveraGen BioPharma. LSC and JNvdA own stock options of ReveraGen. EPH is a co-founder of ReveraGen and owns founder shares.

\section{ESDPPP 2019}

\section{Poster Presentations}

\section{P01 APPLICATION OF FETO-PLACENTAL-MATERNAL PHYSIOLOGICALLY-BASED PHARMACOKINETIC MODEL TO PREDICT TENOFOVIR CONCENTRATION DURING PREGNANCY}

K Abduljalil, TN Johnson, M Jamei. Certara UK Limited, Simcyp Division, Sheffield, UK

\subsection{6/archdischild-2019-esdppp.40}

Background Tenofovir is a drug used in combination with other anti-HIV drugs to treat patients with HIV-1 infection. It is used during pregnancy to reduce the risk of HIV transmission to the child. The aim of this work is to use a Physiologically-Based Pharmacokinetic (PBPK) model for prediction of maternal and fetal tenofovir concentration at birth.

Methods A full Feto-Placental-Maternal PBPK model that includes placenta as a 3-comparment permeability limited organ and 14 compartments for different fetal organs was developed using physiological ${ }^{1,2}$ and drug specific parameters ${ }^{3}$ 